\title{
Adaptive Kernel Estimation of the Conditional Quantiles
}

\author{
Raid B. Salha ${ }^{1}$, Hazem I. El Shekh Ahmed ${ }^{2}$, Hossam O. EL-Sayed ${ }^{3}$
}

${ }^{1}$ The Islamic University of Gaza, Department of Mathematics, Palestine

${ }^{2}$ Al Quds Open University, Department of Mathematics, Palestine

${ }^{3}$ UNRWA, Department of Education, Palestine

Correspondence: Hazem I. El Shekh Ahmed, Al Quds Open University, Department of Mathematics, Palestine. E-mail: hshaikhahmad@qou.edu

$\begin{array}{ll}\text { Received: November 1, } 2015 & \text { Accepted: December 15, } 2015 \quad \text { Online Published: December 23, } 2015 \\ \text { doi:10.5539/ijsp.v5n1p79 } & \text { URL: http://dx.doi.org/10.5539/ijsp.v5n1p79 }\end{array}$

\begin{abstract}
In this paper, we define the adaptive kernel estimation of the conditional distribution function (cdf) for independent and identically distributed (iid) data using varying bandwidth. The bias, variance and the mean squared error of the proposed estimator are investigated. Moreover, the asymptotic normality of the proposed estimator is investigated.
\end{abstract}

The results of the simulation study show that the adaptive kernel estimation of the conditional quantiles with varying bandwidth have better performance than the kernel estimations with fixed bandwidth.

Keywords: Quantile regression, conditional distribution, kernel estimation, asymptotic normality

\section{Introduction}

Suppose $\left\{\left(X_{i}, Y_{i}\right)\right\}_{i=1}^{n}$ are $\mathbf{R} \times \mathbf{R}$ random variables and we assume that the regression model defined by $Y_{i}=m\left(X_{i}\right)+\epsilon_{i}, i=$ $1,2, \ldots n$. We assume that the response variable $Y$ depends on an independent random variable $X$ with common probability density function $f$ and $m(x)$ is the conditional mean curve

$$
m(x)=E(Y \mid X=x)=\int \frac{y f(x, y)}{f(x)} d y,
$$

and a nonparametric kernel estimation of the regression function can be obtained as

$$
\hat{f}(x)=\frac{1}{n h} \sum_{i=1}^{n} K\left(\frac{x-X_{i}}{h}\right) .
$$

Silverman (1986) gave an algorithm with three steps for adaptive kernel estimation of density function. At the first, a prior kernel estimator with a fixed bandwidth is obtained. The second step, the local bandwidth factor $\lambda_{i}$ is defined by

$$
\lambda_{i}=\left\{\hat{f}\left(X_{i}\right) / g\right\}^{-\beta}, \quad 0 \leq \beta \leq 1,
$$

where $g$ (assuming $g \neq 0$ ) is the geometric mean of $\hat{f}\left(X_{i}\right)$. Finally, for one variable the conditional kernel estimation is given by

$$
f_{n}(x)=\frac{1}{n} \sum_{i=1}^{n} \frac{1}{h \lambda_{i}} K\left(\frac{x-X_{i}}{h \lambda_{i}}\right) .
$$

Salha (2009) discussed the same method for the adaptive estimation of the hazard rate function. Salha and El Shekh Ahamed (2009) also discussed the same method for the adaptive estimation of the conditional mode.

The adaptive kernel estimation is equivalent to the kernel estimation with fixed bandwidth when $\beta$ is equal to 0 . When $\beta=1$, then the adaptive kernel estimation is equivalent to the nearest neighbor estimation.

Demir, Toktamis (2010) gave an algorithm to adaptive kernel estimation of density function by setting $g=\sum_{i=1}^{n} \hat{f}\left(X_{i}\right) / n$.

The conditional distribution function has an important role for quantile regression. Yu and Jones (1998), Hall et al. (1999), Salha (2006) and Dette (2011) have recently considered methods for estimating conditional distributions. Yu (2003) discussed some problems for estimation of the conditional quantiles. 
Abberger (1997) defined a kernel estimation of the conditional distribution function as

$$
\hat{F}(y \mid x)=\frac{\sum_{i=1}^{n} I_{\left\{Y_{i} \leqslant y\right\}} K\left(\frac{x-X_{i}}{h}\right)}{\sum_{i=1}^{n} K\left(\frac{x-X_{i}}{h}\right)},
$$

and the conditional $\alpha$ - quantile is given by

$$
\hat{q}_{\alpha}(x)=\inf \{y \in R \mid \hat{F}(y \mid x) \geq \alpha\}, \quad 0<\alpha<1 .
$$

Abberger (1997) discussed the bias, variance and the mean squared error of this estimator, and proved that the asymptotic normality of the proposed estimator.

From the previous, we have noticed a lot of statisticians use the kernel estimation of the density and conditional distribution function, but a question is raised: Is there any adjustments or improvements to this estimated whose gives better results? In this paper, we will use the adaptive kernel estimation of the conditional distribution function to estimate the conditional quantiles.

We proposed the following estimator of the conditional distribution function as

$$
F_{n}(y \mid x)=\frac{\sum_{i=1}^{n} \frac{1}{h \lambda_{i}} I_{\left\{Y_{i} \leqslant y\right\}} K\left(\frac{x-X_{i}}{h \lambda_{i}}\right)}{\sum_{i=1}^{n} \frac{1}{h \lambda_{i}} K\left(\frac{x-X_{i}}{h \lambda_{i}}\right)},
$$

where we define the local bandwidth factor $\lambda_{i}$ by

$$
\lambda_{i}=\left[\frac{f_{n}\left(X_{i}\right)}{g}\right]^{-\beta}, \quad 0 \leq \beta \leq 1,
$$

where $\mathrm{g}$ is the arithmetic mean of $f_{n}\left(X_{i}\right)$.

Now, we defined the adaptive of the conditional $\alpha$ - quantile is given by

$$
q_{n, \alpha}(x)=\inf \left\{y \in R \mid F_{n}(y \mid x) \geq \alpha\right\}, \quad 0<\alpha<1 .
$$

\section{Preliminaries}

In this section, we will consider an assumptions of the kernel function, the bandwidth and the conditional distribution function. These assumptions are:

(A1) $h=h_{n}$ is sequence of positive number satisfies the following:

(i) $\quad h \longrightarrow 0$, for $n \longrightarrow \infty$;

(ii) $\quad n h \longrightarrow \infty$, for $n \longrightarrow \infty$;

(A2) The kernel $K$ is a Borel function and satisfies the following:

(i) $K$ has compact support;

(ii) $\quad K$ is symmetric;

(iii) $\quad K$ is Lipschitz-continuous;

(iv) $\int K(u) d u=1$; 
(v) $K$ is bounded;

(A3) For fixed $y \in \mathbb{R}$ there exists $F^{\prime \prime}(y \mid x)=\frac{\partial^{2} F(y \mid x)}{\partial x^{2}}$ in a neighborhood of $x$.

Now, we state and prove some lemmas that will help us to achieve the main result in this paper:

Lemma 1 For any random variable $X$ with density $f$, we have

$$
E\left[f_{n}(x)\right]-f(x)=\frac{1}{2} h^{2} \mu_{2}(x) f^{\prime \prime}(x)+o(h)^{2} .
$$

Proof:

Assume that $\int_{-\infty}^{\infty} K(u) d u=1$, then we have $\int_{-\infty}^{\infty} u K(u) d u=0$.

And we assume that $\mu_{2}(x)=\int_{-\infty}^{\infty} u^{2} K(u) d u<\infty$.

$$
\begin{aligned}
E\left[f_{n}(x)\right] & =E\left[\frac{1}{n h} \sum_{i=1}^{n} K\left(\frac{x-X_{i}}{h}\right)\right] \\
& =\frac{1}{h} \int_{-\infty}^{\infty} K\left(\frac{x-X_{i}}{h}\right) f(y) d y
\end{aligned}
$$

Let $\frac{x-y}{h}=z, \quad$ then $y=x-h z$, and so

$$
\begin{aligned}
E\left[f_{n}(x)\right] & =\frac{1}{n h} \sum_{i=1}^{n} \int_{-\infty}^{\infty} K(z) f(x-z h) h d z \\
& =\int_{-\infty}^{\infty} K(z) f(x-z h) d z
\end{aligned}
$$

Now, using Taylors expansion for $f(x-z h)$ to get

$$
f(x-z h)=f(x)-z h f^{\prime}(x)+\frac{(z h)^{2}}{2} f^{\prime \prime}(x)+o\left(h^{2}\right)
$$

Now,

$$
\begin{aligned}
E\left[f_{n}(x)\right] & =\int_{-\infty}^{\infty} K(z)\left[f(x)-z h f^{\prime}(x)+\frac{(z h)^{2}}{2} f^{\prime \prime}(x)+o\left(h^{2}\right)\right] d z \\
& =\int_{-\infty}^{\infty} K(z) f(x) d z-h f^{\prime}(x) \int_{-\infty}^{\infty} z K(z) d z+\frac{h^{2}}{2} f^{\prime \prime}(x) \int_{-\infty}^{\infty} z^{2} K(z) d z \\
& +\int_{-\infty}^{\infty} o\left(h^{2}\right) K(z) d z \\
& =f(x)+\frac{1}{2} h^{2} \mu_{2}(x) f^{\prime \prime}(x)+o(h)^{2} .
\end{aligned}
$$

Thus, we have the result.

Lemma 2 ( Integral approximation of the sum over the kernel function )

With (A2)(i), Lipschitz-continuity, (A2)(iii), $U_{i}(x)=\frac{x-X_{i}}{h}, x \in(h, 1-h)$ and the mean value theorem of integration, it follows:

1. $\lim _{n \rightarrow \infty} \sum_{i=1}^{n} \frac{1}{n h} K\left(U_{i}\right)=\int_{-\infty}^{\infty} K(u) d u$

2. $\lim _{n \rightarrow \infty} \sum_{i=1}^{n} \frac{1}{n h} K^{2}\left(U_{i}\right)=\int_{-\infty}^{\infty} K^{2}(u) d u$ 
3. $\lim _{n \rightarrow \infty} \sum_{i=1}^{n} \frac{1}{n h} U_{i} K\left(U_{i}\right)=\int_{-\infty}^{\infty} u K(u) d u$

Proof: See Abberger (1997).

\section{Bias, Variance and Mean Squared Error}

In this section, the bias and variance will be evaluated. For independent $\left\{Y_{i}\right\}$ the expectation and the variance of the estimator $F_{n}(y \mid x)$ are follows as:

$$
E\left[F_{n}(y \mid x)\right]=\sum_{i=1}^{n} \frac{K\left(\frac{x-X_{i}}{h \lambda_{i}}\right)}{\left[\sum_{i=1}^{n} K\left(\frac{x-X_{i}}{h \lambda_{i}}\right)\right]} F\left(y \mid X_{i}\right)
$$

and

$$
\operatorname{Var}\left[F_{n}(y \mid x)\right]=\sum_{i=1}^{n} \frac{K^{2}\left(\frac{x-X_{i}}{h \lambda_{i}}\right)}{\left[\sum_{i=1}^{n} K\left(\frac{x-X_{i}}{h \lambda_{i}}\right)\right]^{2}}\left[F\left(y \mid X_{i}\right)-F^{2}\left(y \mid X_{i}\right)\right] .
$$

\subsection{The Mean Squared Error}

In this subsection, we will introduce the definition of the mean squared error and give the main theorem about the asymptotic mean squared error.

The mean squared error (MSE) is used to measure the error when estimating the density function at a single point. It is defined by

$$
\operatorname{MSE}\left\{f_{n}(x)\right\}=E\left\{f_{n}(x)-f(x)\right\}^{2}
$$

That is, equation (4) can be written as

$$
M S E\left\{f_{n}(x)\right\}=\left(E f_{n}(x)-f(x)\right)^{2}+\operatorname{Var} f_{n}(x)
$$

Theorem 1 Let $\left\{Y_{i}\right\}$ be independent and let (A1)(i,ii), (A2)( i, ii, iii, iv) and (A3) be satisfied. Then it holds for $n \longrightarrow \infty$

$$
\begin{aligned}
\operatorname{MSE}\left(F_{n}(y \mid x)\right) & \approx\left[\frac{\left(h \lambda_{i}\right)^{2}}{2} F^{\prime \prime}(y \mid x) \int u^{2} K(u) d u\right]^{2} \\
& +\frac{1}{n\left(h \lambda_{i}\right)}\left(F(y \mid x)-F^{2}(y \mid x)\right) \int K^{2}(u) d u .
\end{aligned}
$$

Proof:

By equation (5) and equation (1) the bias term is hold.

That is $\quad\left[E\left[F_{n}(x)\right]-F(x)\right]^{2}=\left[\frac{\left(h \lambda_{i}\right)^{2}}{2} F^{\prime \prime}(y \mid x) \int u^{2} K(u) d u\right]^{2}$

we only want to find $\operatorname{Var}\left[F_{n}(y \mid x)\right]$.

Using Taylors expansion for $F\left(y \mid X_{i}\right)$ and $F^{2}\left(y \mid X_{i}\right)$ to get

$$
\begin{aligned}
F\left(y \mid x-h \lambda_{i} U_{i}\right) & =F(y \mid x)-h \lambda_{i} U_{i} F^{\prime}(y \mid x)+\left(h \lambda_{i}\right)^{2} U_{i}^{2} F^{\prime \prime}(y \mid x)+o\left(h \lambda_{i}\right)^{2}, \\
F^{2}\left(y \mid x-h \lambda_{i} U_{i}\right) & =F^{2}(y \mid x)+\left(-h \lambda_{i} U_{i}\right)\left[F^{2}(y \mid x)\right]^{\prime}+\left(-h \lambda_{i} U_{i}\right)^{2}\left[F^{2}(y \mid x)\right]^{\prime \prime}+o\left(h \lambda_{i}\right)^{2} \\
& =F^{2}(y \mid x)-2 h \lambda_{i} U_{i} F(y \mid x) F^{\prime}(y \mid x)+2\left(h \lambda_{i}\right)^{2} U_{i}^{2} F^{\prime 2}(y \mid x) \\
& +2\left(h \lambda_{i}\right)^{2} U_{i}^{2} F(y \mid x) F^{\prime \prime}(y \mid x)+o\left(h \lambda_{i}\right)^{2},
\end{aligned}
$$


Then $F\left(y \mid x-h \lambda_{i} U_{i}\right)-F^{2}\left(y \mid x-h \lambda_{i} U_{i}\right)=$

$$
\begin{aligned}
& =F(y \mid x)-h \lambda_{i} U_{i} F^{\prime}(y \mid x)+\left(h \lambda_{i}\right)^{2} U_{i F^{\prime \prime}(y \mid x)}^{2} F^{\prime \prime}(y \mid x) \\
& -F^{2}(y \mid x)+2 h \lambda_{i} U_{i} F(y \mid x) F^{\prime}(y \mid x)-2\left(h \lambda_{i}\right)^{2} U_{i}^{2} F^{\prime 2}(y \mid x) \\
& -2\left(h \lambda_{i}\right)^{2} U_{i}^{2} F(y \mid x) F^{\prime \prime}(y \mid x) \\
& =\left[F(y \mid x)-F^{2}(y \mid x)\right]+h \lambda_{i} U_{i}\left[-F^{\prime}(y \mid x)+2 F(y \mid x) F^{\prime}(y \mid x)\right] \\
& +\left(h \lambda_{i}\right)^{2} U_{i}^{2}\left[\left(h \lambda_{i}\right)^{2} U_{i}^{2}-2 F(y \mid x) F^{\prime \prime}(y \mid x)-2 F^{2}(y \mid x)\right]
\end{aligned}
$$

As $\quad n \rightarrow \infty$, and $h \rightarrow 0$, we have $h^{2} \rightarrow 0$.

That is $F\left(y \mid X_{i}\right)-F^{2}\left(y \mid X_{i}\right) \approx F(y \mid x)-F^{2}(y \mid x)$, so we have

$$
\begin{aligned}
\operatorname{Var}\left[F_{n}(y \mid x)\right. & =\frac{\sum_{i=1}^{n} K^{2}\left(\frac{x-X_{i}}{h \lambda_{i}}\right)}{\left[\sum_{i=1}^{n} K\left(\frac{x-X_{i}}{h \lambda_{i}}\right)\right]^{2}}\left[F(y \mid x)-F^{2}(y \mid x)\right] \\
& =\frac{\left(n h \lambda_{i}\right)}{\left(n h \lambda_{i}\right)^{2}}\left[F(y \mid x)-F^{2}(y \mid x)\right] \int_{-\infty}^{\infty} K^{2}(u) d u \\
& =\frac{1}{\left(n h \lambda_{i}\right)}\left[F(y \mid x)-F^{2}(y \mid x)\right] \int_{-\infty}^{\infty} K^{2}(u) d u
\end{aligned}
$$

Thus, we have the result.

\section{Asymptotic Normality}

In this section, the next theorems show the asymptotic normality $\left(n h \lambda_{i}\right)^{\frac{1}{2}}\left(F_{n}(y \mid x)-E\left[F_{n}(y \mid x)\right]\right)$ and $\left(n h \lambda_{i}\right)^{\frac{1}{2}}\left(F_{n}(y \mid x)-\right.$ $[F(y \mid x)])$.

Theorem 2 Let the condition of the last theorem be satisfied. Then it holds for $n \longrightarrow \infty$,

$$
\left(n h \lambda_{i}\right)^{\frac{1}{2}}\left(F_{n}(y \mid x)-E\left[F_{n}(y \mid x)\right]\right) \stackrel{d}{\longrightarrow} N\left(0,\left[F(y \mid x)-F^{2}(y \mid x)\right] \int K^{2}(u) d u\right) .
$$

Proof:

To prove this theorem, we use Liapunov's condition. So let

$$
Q_{n, i}(x)=\frac{\frac{K\left(\frac{x-X_{i}}{h \lambda_{i}}\right)}{\sum_{i=1}^{n} K\left(\frac{x-X_{i}}{h \lambda_{i}}\right)}\left[I_{\left\{Y_{i} \leq y\right\}}-F\left(y \mid X_{i}\right)\right]}{\sqrt{\operatorname{Var}\left[F_{n}(y \mid x)\right]}}
$$

Therefor,

$$
\sum_{i=1}^{n} Q_{n, i}(x)=\sum_{i=1}^{n} \frac{\frac{K\left(\frac{x-X_{i}}{h \lambda_{i}}\right)}{\sum_{i=1}^{n} K\left(\frac{x-X_{i}}{h \lambda_{i}}\right)}\left[I_{\left\{Y_{i} \leq y\right\}}-F\left(y \mid X_{i}\right)\right]}{\sqrt{\operatorname{Var}\left[F_{n}(y \mid x)\right]}}
$$

That is

This means that

$$
\sum_{i=1}^{n} Q_{n, i}(x)=\sum_{i=1}^{n} \frac{\frac{K\left(\frac{x-X_{i}}{h \lambda_{i}}\right)}{\sum_{i=1}^{n} K\left(\frac{x-X_{i}}{h \lambda_{i}}\right)} I_{\left\{Y_{i} \leq y\right\}}-\sum_{i=1}^{n} \frac{K\left(\frac{x-X_{i}}{h \lambda_{i}}\right)}{\sum_{i=1}^{n} K\left(\frac{x-X_{i}}{h \lambda_{i}}\right)} F\left(y \mid X_{i}\right)}{\sqrt{\operatorname{Var}\left[F_{n}(y \mid x)\right]}}
$$




$$
\sum_{i=1}^{n} Q_{n, i}(x)=\frac{F_{n}(y \mid x)-E\left[F_{n}(y \mid x)\right]}{\sqrt{\operatorname{Var}\left[F_{n}(y \mid x)\right]}}
$$

Now, the Liapunov's condition

$$
\lim _{n \rightarrow \infty} \sum_{i=1}^{\infty} E\left|Q_{n, i}(x)\right|^{3}=\lim _{n \rightarrow \infty} \frac{\sum_{i=1}^{n} E\left|\frac{K\left(\frac{x-X_{i}}{h \lambda_{i}}\right)}{\sum_{i=1}^{n} K\left(\frac{x-X_{i}}{h \lambda_{i}}\right)}\left[I_{\left\{Y_{i} \leq y\right\}}-F\left(y \mid X_{i}\right)\right]\right|^{3}}{\left(\operatorname{Var}\left[F_{n}(y \mid x)\right]\right)^{\frac{3}{2}}}
$$

The numerator is $\sum_{i=1}^{n} E\left|\frac{K\left(\frac{x-X_{i}}{h \lambda_{i}}\right)}{\sum_{i=1}^{n} K\left(\frac{x-X_{i}}{h \lambda_{i}}\right)}\left[I_{\left\{Y_{i} \leq y\right\}}-F\left(y \mid X_{i}\right)\right]\right|^{3}$

$$
\begin{aligned}
& =\sum_{i=1}^{n} E\left\{\left|\frac{K\left(\frac{x-X_{i}}{h \lambda_{i}}\right)}{\sum_{i=1}^{n} K\left(\frac{x-X_{i}}{h \lambda_{i}}\right)}\right|^{3}\left|\left[I_{\left\{Y_{i} \leq y\right\}}-F\left(y \mid X_{i}\right)\right]\right|^{3}\right\} \\
& =\sum_{i=1}^{n}\left\{\left|\frac{K\left(\frac{x-X_{i}}{h \lambda_{i}}\right)}{\sum_{i=1}^{n} K\left(\frac{x-X_{i}}{h \lambda_{i}}\right)}\right|^{3} E\left|\left[I_{\left\{Y_{i} \leq y\right\}}-F\left(y \mid X_{i}\right)\right]\right|^{3}\right\} \\
& \leq \sum_{i=1}^{n}\left\{\left|\frac{K\left(\frac{x-X_{i}}{h \lambda_{i}}\right)}{\sum_{i=1}^{n} K\left(\frac{x-X_{i}}{h \lambda_{i}}\right)}\right|^{3}\right\}=\frac{\sum_{i=1}^{n} K^{3}\left(\frac{x-X_{i}}{h \lambda_{i}}\right)}{\left[\sum_{i=1}^{n} K\left(\frac{x-X_{i}}{h \lambda_{i}}\right)\right]^{3}} \\
& =\frac{n h \lambda_{i} \int K\left(\frac{x-X_{i}}{h \lambda_{i}}\right) d u}{\left[n h \lambda_{i} \int K\left(\frac{x-X_{i}}{h \lambda_{i}}\right) d u\right]^{3}}=\frac{o\left(n h \lambda_{i}\right)}{o\left(n^{3} h \lambda_{i}^{3}\right)}=o\left(\frac{1}{n^{2} h \lambda_{i}^{2}}\right)
\end{aligned}
$$

But, the variance of $F_{n}(y \mid x)$ follows as $\operatorname{Var}\left[F_{n}(y \mid x)\right]=o\left(\frac{1}{n h \lambda_{i}}\right)$

That is

$$
\left(\operatorname{Var}\left[F_{n}(y \mid x)\right]\right)^{\frac{3}{2}}=o\left(\frac{1}{n^{\frac{3}{2}} h \lambda_{i}^{\frac{3}{2}}}\right)
$$

Then

$$
\lim _{n \rightarrow \infty} \sum_{i} E\left|Q_{n, i}(x)\right|^{3} \leq \frac{O\left(\frac{1}{n^{2} h \lambda_{i}^{2}}\right)}{O\left(\frac{1}{n^{\frac{3}{2}} h \lambda_{i}^{\frac{3}{2}}}\right)}=O\left(\frac{1}{n^{\frac{1}{2}} h \lambda_{i}^{\frac{1}{2}}}\right)=o(1) .
$$

That is the Liapunov's condition holds. Then

$$
\frac{F_{n}(y \mid x)-E\left[F_{n}(y \mid x)\right]}{\sqrt{\operatorname{Var}\left[F_{n}(y \mid x)\right]}} \rightarrow N(0,1)
$$

Thus

$$
\left(n h \lambda_{i}\right)^{\frac{1}{2}}\left(F_{n}(y \mid x)-E\left[F_{n}(y \mid x)\right]\right) \stackrel{d}{\longrightarrow} N\left(0,\left[F(y \mid x)-F^{2}(y \mid x)\right] \int K^{2}(u) d u\right) .
$$


Corollary 1 Let the condition of Theorem 1 be satisfied, and $n\left(h \lambda_{i}\right)^{5} \longrightarrow 0$. Then it holds for $n \longrightarrow \infty$,

$$
\left(n h \lambda_{i}\right)^{\frac{1}{2}}\left(F_{n}(y \mid x)-F(y \mid x)\right) \stackrel{d}{\longrightarrow} N\left(0,\left[F_{n}(y \mid x)-F^{2}(y \mid x)\right] \int K^{2}(u) d u\right) .
$$

Proof:

Since $\left(n h \lambda_{i}\right)^{\frac{1}{2}}\left(F_{n}(y \mid x)-E\left[F_{n}(y \mid x)\right]\right) \stackrel{d}{\longrightarrow} N\left(0,\left[F(y \mid x)-F^{2}(y \mid x)\right] \int K^{2}(u) d u\right)$.

and

$$
\begin{aligned}
E\left[F_{n}(y \mid x)\right]-F(y \mid x) & =\frac{1}{2}\left(h \lambda_{i}\right)^{2} \int_{\infty}^{\infty} u^{2} K(u) d u F^{\prime \prime}(x)+o\left(h \lambda_{i}\right)^{2} \\
& =O\left(h \lambda_{i}\right)^{2}
\end{aligned}
$$

Then

$$
\begin{aligned}
\left(n h \lambda_{i}\right)^{\frac{1}{2}} E\left[F_{n}(y \mid x)\right]-F(y \mid x) & =\left(n h \lambda_{i}\right)^{\frac{1}{2}} \cdot O\left(h \lambda_{i}\right)^{2} \\
& =O\left(n\left[h \lambda_{i}\right]^{5}\right)^{\frac{1}{2}}
\end{aligned}
$$

With $n[h \lambda]_{i}^{5} \rightarrow 0$, we have $E\left[F_{n}(y \mid x)\right] \rightarrow F(y \mid x)$

That is

$$
\left(n h \lambda_{i}\right)^{\frac{1}{2}}\left(F_{n}(y \mid x)-F(y \mid x)\right) \stackrel{d}{\longrightarrow} N\left(0,\left[F_{n}(y \mid x)-F^{2}(y \mid x)\right] \int K^{2}(u) d u\right) .
$$

Now, let

$$
H_{n, \alpha}(\theta(x))=\sum_{i=1}^{n} \frac{1}{\sum_{i=1}^{n} K\left(\frac{x-X_{i}}{h \lambda_{i}}\right)} \cdot K\left(\frac{x-X_{i}}{h \lambda_{i}}\right)\left[\alpha-I_{\left\{Y_{i} \leq \theta(x)\right\}}\right]
$$

Using the central limit theorem, we have

$$
\frac{H_{n, \alpha}(\theta(x))-E\left[H_{n, \alpha}(\theta(x))\right]}{\sqrt{\operatorname{Var}\left[H_{n, \alpha}(\theta(x))\right]}} \stackrel{d}{\longrightarrow} N(0,1), \quad n \longrightarrow \infty .
$$

With $H_{n, \alpha}(\theta(x))$ the mean squared error of $q_{n, \alpha}(x)$ can be calculated.

Theorem 3 Let the conditions of Theorem 1 be satisfied and let $F_{n}\left(q_{n, \alpha}(x) \mid x\right)=F\left(q_{\alpha}(x) \mid x\right)=\alpha$ be unique. Then it holds

$$
\begin{aligned}
\operatorname{MSE}\left[q_{n, \alpha}(x)\right] & =\left[\frac{1}{2}(h \lambda)_{i}^{2} \frac{F^{\prime \prime}\left(q_{\alpha}(x) \mid x\right)}{f\left(q_{\alpha}(x) \mid x\right)} \int u^{2} K(u) d u\right]^{2} \\
& +\frac{1}{n h \lambda_{i}} \frac{\alpha(1-\alpha)}{f^{2}\left(q_{\alpha}(x)\right)} \int K^{2}(u) d u
\end{aligned}
$$

Proof:

By the Taylor expansion of the conditional distribution functions of theorem (3.1.1) and $\theta(x)=q_{n, \alpha}(x)$ follows

$$
\begin{aligned}
E\left[H_{n, \alpha}\left(q_{n, \alpha}(x)\right)\right] & \approx f\left(q_{\alpha}(x) \mid x\right)\left[q_{n, \alpha}(x)-q_{\alpha}(x)\right] \\
& +\frac{1}{2}\left(h \lambda_{i}\right)_{n}^{2} F^{\prime \prime}\left(q_{\alpha}(x)\right) \frac{\sum_{i=1}^{n} U_{i}^{2} K\left(U_{i}\right)}{\sum_{i=1}^{n} K\left(U_{i}\right)}
\end{aligned}
$$

and with integral approximation holds

$$
\begin{aligned}
E\left[H_{n, \alpha}\left(q_{n, \alpha}(x)\right)\right] & \approx f\left(q_{\alpha}(x) \mid x\right)\left[q_{n, \alpha}(x)-q_{\alpha}(x)\right] \\
& +\frac{1}{2}(h \lambda)_{n}^{2} F^{\prime \prime}\left(q_{\alpha}(x)\right) \int_{-\infty}^{\infty} u^{2} K(u) d u .
\end{aligned}
$$


Now,

$$
\begin{aligned}
\operatorname{Var}\left[H_{n, \alpha}\left(q_{n, \alpha}(x)\right)\right] & =\frac{1}{\left[\sum_{i=1}^{n} K\left(U_{i}\right)\right]^{2}} \sum_{i=1}^{n} K^{2}\left(U_{i}\right)\left[F\left(q_{n, \alpha}(x) \mid x-h \lambda_{i} U_{i}\right)\right. \\
& \left.-F^{2}\left(\left(q_{n, \alpha}(x)\right) \mid x-h \lambda_{i} U_{i}\right)\right] \\
& \approx \frac{1}{\left[\sum_{i=1}^{n} K\left(U_{i}\right)\right]^{2}}[\alpha(1-\alpha)] \sum_{i=1}^{n} K^{2}\left(U_{i}\right) \\
& \approx \frac{1}{n h \lambda_{i}} \alpha(1-\alpha) \int K^{2}(u) d u .
\end{aligned}
$$

This implies for $n \longrightarrow \infty$

$$
\frac{f\left(q_{\alpha}(x) \mid x\right)\left[q_{n, \alpha}(x)-q_{\alpha}(x)\right]+\frac{1}{2} h \lambda_{i}^{2} F^{\prime \prime}\left(q_{\alpha}(x)\right) \int_{-\infty}^{\infty} u^{2} K(u) d u}{\sqrt{\frac{1}{n h \lambda_{i}} \alpha(1-\alpha) \int_{-\infty}^{\infty} K^{2}(u) d u}} \rightarrow N(0,1) .
$$

Corollary 2 Let the condition of Theorem 1 be satisfied and let $n h_{n}^{5} \longrightarrow 0$, for $n \longrightarrow \infty$.

Then it holds

$$
\begin{aligned}
\left(n h \lambda_{i}\right)^{\frac{1}{2}}\left(q_{n, \alpha}(x)-q_{\alpha}(x)\right) \stackrel{d}{\longrightarrow} & N\left(\frac{1}{2}\left(n h \lambda_{i}^{5}\right)^{\frac{1}{2}} \frac{F^{\prime \prime}\left(q_{\alpha}(x) \mid x\right)}{f\left(q_{\alpha}(x) \mid x\right)} \int_{-\infty}^{\infty} u^{2} K(u) d u,\right. \\
& \left.\frac{\alpha(1-\alpha)}{\left.f^{2}\left(q_{\alpha}(x) \mid x\right)\right)} \int_{-\infty}^{\infty} K^{2}(u) d u\right) ; \\
\stackrel{d}{\longrightarrow} & N\left(0, \frac{\alpha(1-\alpha)}{f^{2}\left(q_{\alpha}(x) \mid x\right)} \int_{-\infty}^{\infty} K^{2}(u) d u\right) .
\end{aligned}
$$

\section{Simulation Studies}

In this section, two simulation studies were conducted to compare the performance of the KRE estimation of the conditional distribution function and the AKRE estimation of the conditional distribution function.

In this studies, 400 data points are simulated from $\mathrm{R}$ program.

\subsection{Case Study 1}

We generated samples using R program of size $25,50,75,150,250,400,700$ from the regression function $y=\cos x+e x$, where the $x_{i}$ were drawn from a uniform distribution based on the interval $[0,1]$, and the $e$ have a normal distribution $N(0,1)$.

The fixed bandwidth $h$ was computed by Silverman (1986) and Wand and Jones (1995) as

$$
h_{\text {opt }}=1.06 \mathrm{sd}(\mathrm{t}) n^{-\frac{1}{5}} \text {. }
$$

Now, we define a pilot estimate $f_{n}\left(X_{i}\right)$ that satisfies $f_{n}\left(X_{i}\right) \geq 0$, where $\left\{X_{i}\right\}$ is a family of iid. In this paper we select the Epanechnikov kernel

$$
K(t)=\frac{3}{4}\left(1-\frac{1}{5} t^{2}\right) / \sqrt{5} \quad \text { for }|t|<\sqrt{5} .
$$

We define the local bandwidth factor $\lambda_{i}$ by

$$
\lambda_{i}=\left[\frac{f_{n}\left(X_{i}\right)}{g}\right]^{-\frac{1}{2}}
$$

where $\mathrm{g}$ is the arithmetic mean of $f_{n}\left(X_{i}\right)$. We have considered that the equation

$$
F_{n}(y \mid x)=\frac{\sum_{i=1}^{n} \frac{1}{h \lambda_{i}} K\left(\frac{x-X_{i}}{h \lambda_{i}}\right) I_{\left\{Y_{i} \leqslant y\right\}}}{\sum_{i=1}^{n} \frac{1}{h \lambda_{i}} K\left(\frac{x-X_{i}}{h \lambda_{i}}\right)}
$$


The graph of the real regression function of the estimation of the regression function and the adaptive regression function.

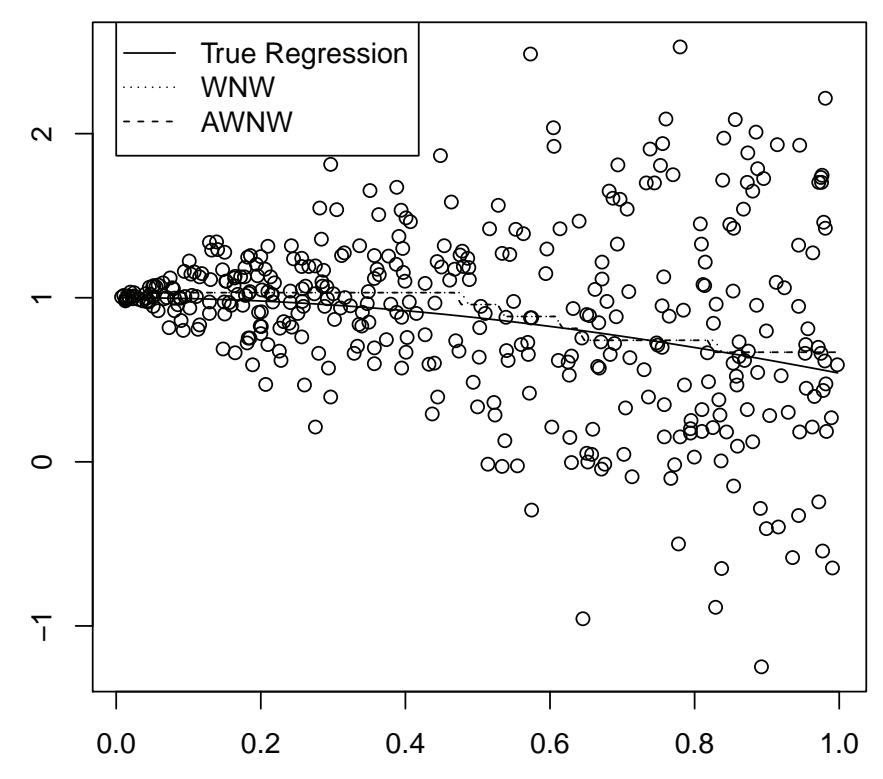

Figure 1: Improved Kernel Quantile Regression Estimator at $n=75$

Figure 1. shows the plot of the data together which the true regression curve, the kernel quantile regression estimator and the improved kernel quantile regression estimator (This graph at $n=75$ ). The continuous line indicates that the kernel regression, the dotted line indicates that the kernel regression estimator and the intermittent line indicates that the improvement kernel regression estimator.

The mean square error was computed as

Table 1. MSE and MSE Adaptive for samples

\begin{tabular}{lcc}
\hline $\mathrm{n}$ & MSE & MSE Adaptive \\
\hline 25 & 0.023159180 & $0.022495930^{*}$ \\
50 & 0.009156496 & $0.009156385^{*}$ \\
75 & 0.004575978 & $0.004380828^{*}$ \\
150 & 0.004350594 & $0.004143210^{*}$ \\
250 & 0.003715318 & $0.003494345^{*}$ \\
400 & 0.003178363 & $0.003001811^{*}$ \\
700 & 0.002912181 & $0.002770896^{*}$ \\
\hline
\end{tabular}

* Minimum MSE in each row.

As seen from Table 1, for all sample sizes, we have the kernel estimators using varying bandwidths have smaller MSE values than the kernel estimator with fixed bandwidth. In each case, it is seen that MSE has the best performance.

\subsection{Case Study 2}

Another study was conducted to compare the performance of the KRE estimation of the conditional distribution function and the AKRE estimation of the conational distribution function.

We generated samples using $\mathrm{R}$ program of size $25,50,75,150,250,400,700$ from the regression function $y=1-x+$ 
$3 e^{-200 x}+e x$, where the $x_{i}$ were drawn from a uniform distribution based on the interval [0,1], and the $e$ have a normal distribution $N(0,1)$ and $h, \lambda_{i}$ computed as the last simulation.

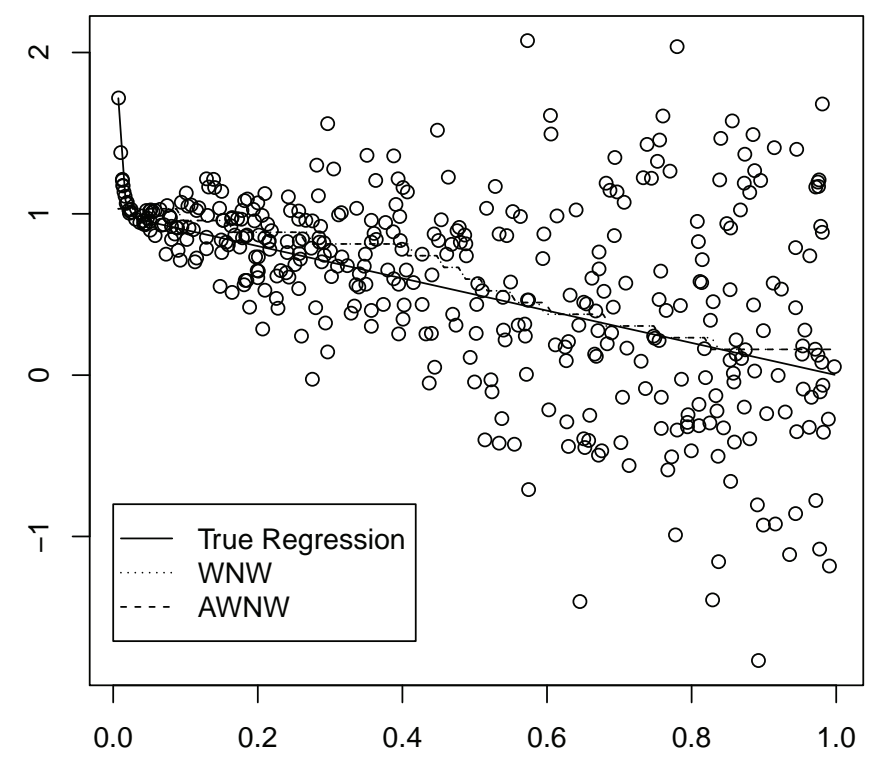

Figure 2: Improved Kernel Quantile Regression Estimator at $\mathrm{n}=75$

Figure 2. shows the plot of the data together which the true regression curve, the kernel quantile regression estimator and the improved kernel quantile regression estimator (This graph at $n=75$ ). The continuous line indicates that the kernel regression, the dotted line indicates that the kernel regression estimator and the intermittent line indicates that the improvement kernel regression estimator.

The mean square error was computed as

Table 2. MSE and MSE Adaptive for samples

\begin{tabular}{lcc}
\hline $\mathrm{n}$ & MSE & MSE Adaptive \\
\hline 25 & 0.03620139 & $0.03594952^{*}$ \\
50 & 0.01819106 & $0.01790818^{*}$ \\
75 & 0.01451794 & $0.01408981^{*}$ \\
150 & 0.01170675 & $0.01113603^{*}$ \\
250 & 0.01083405 & $0.01070306^{*}$ \\
400 & 0.01076291 & $0.01047524^{*}$ \\
700 & 0.01023944 & $0.01002705^{*}$ \\
\hline
\end{tabular}

* Minimum MSE in each row.

\section{Conclusion}

In this paper, we have studied the adaptive kernel estimation of the conditional quantiles and we concluded the expectation, variance, mean square error and asymptotically distribution of the proposed estimator.

The results of the simulation studies showed the adaptive kernel estimation of the conditional quantile with varying bandwidths provide better estimates than the conditional quantile estimate with fixed bandwidth. 


\section{References}

Abberger, K. (1997). Quantile smoothing in financial time series. Statistical Papers, 38, 128-148. http://dx.doi.org/10.1007/BF02925220

Demir, S., \& Toktamis, Ö. (2010). On the adaptive Nadaraya-Watson kernel regression estimation. Hacettepe Journal of Mathimatics and Statistic, 39(3), 429-437

Dette, H., \& Scheder, R. (2011). Estimation of additive quantile regression. Ann Inst Stat Math, 63, 245-265. http://dx.doi.org/10.1007/s10463-009-0225-5

Salha, R. (2006). Kernel estimation of the conditional quantiles and mode for time series. University Of Macedonia.

Salha, R. (2009). Adaptive kernel estimation of the hazard rate1 function. The Islamic University - Gaza Journal, 17(1), 71-81.

Salha, Raid B., \& El Shekh Ahmed, H. (2009). Local variable kernel estimation of the conditional mode. Rajasthan Academy of Physical Sciences, 8(3), 265-280.

Silverman, B. W. (1986). Density estimation for statistics and data analysis. School of Mathematics University of Bath, UK. http://dx.doi.org/10.1007/978-1-4899-3324-9

Wand, M. P., \& Jones, M. C. (1995). Kernel smoothing. University of New South Wales Asutralia. http://dx.doi.org/10.1007/978-1-4899-4493-1

Yu, K. (2003). Quantile regression: Application and current reseach areas. University of Plymouth. UK. http://dx.doi.org/10.1111/1467-9884.00363

Yu, K., \& Jones M. C. (1998). Local linear quantile regression. Journal of the American Statistical Association, 93(441), 228-237. http://dx.doi.org/10.1080/01621459.1998.10474104

\section{Copyrights}

Copyright for this article is retained by the author(s), with first publication rights granted to the journal.

This is an open-access article distributed under the terms and conditions of the Creative Commons Attribution license (http://creativecommons.org/licenses/by/3.0/). 